\title{
Floquet oscillations in periodically driven Dirac systems
}

\author{
Vanessa Junk $\odot$, Phillipp Reck, Cosimo Gorini, and Klaus Richter* \\ Institut für Theoretische Physik, Universität Regensburg, D-93040 Regensburg, Germany
}

(Received 12 June 2019; accepted 9 March 2020; published 2 April 2020)

\begin{abstract}
Electrons in a lattice exhibit time-periodic motion, known as Bloch oscillation, when subject to an additional static electric field. Here, we show that a corresponding dynamics can occur upon replacing the spatially periodic potential by a time-periodic driving: Floquet oscillations of charge carriers in a spatially homogeneous system. The time lattice of the driving gives rise to Floquet bands that take on the role of the usual Bloch bands. For two different drivings (harmonic driving and periodic kicking through pulses) of systems with linear dispersion we demonstrate the existence of such oscillations, both by directly propagating wave packets and based on a complementary Floquet analysis. The Floquet oscillations feature richer oscillation patterns than their Bloch counterpart and enable the imaging of Floquet bands. Moreover, their period can be directly tuned through the driving frequency. Such oscillations should be experimentally observable in effective Dirac systems, such as graphene, when illuminated with circularly polarized light.
\end{abstract}

DOI: 10.1103/PhysRevB.101.134302

\section{INTRODUCTION}

In the early days of quantum mechanics, Bloch and Zener $[1,2]$ predicted that electrons in a periodic potential, when accelerated by a constant external electric field, perform a time-periodic motion, by now well known as Bloch oscillation [3]. It took about 60 years until this phenomenon was observed in biased semiconductor superlattices [4-7]. Since then Bloch oscillations or analogs of them have been found in various systems ranging from cold atom gases $[8,9]$ to classical optical $[10,11]$ and acoustic waves [12], to name a few. In 2014, Bloch oscillations due to the crystal lattice of a biased bulk semiconductor were eventually observed [13].

In the meantime, scientific interest in tuning Bloch bands by means of external time-periodic driving has rapidly grown, especially since the proposal of so-called Floquet topological insulators [14] demonstrating the powerful influence external driving can exert on the properties of a crystal. Recent experiments also showed that Floquet band engineering allows for switching Bloch oscillations on and off [15]. Moreover, additional driving can immensely increase the amplitude of conventional Bloch oscillations, giving rise to "super" Bloch oscillations [16-19].

Here, we propose to consider the opposite limit of a timeperiodically driven system without any spatial lattice, but still subject to a constant external electric field. We demonstrate that, most notably, spatially periodic motion of the charge carriers can still appear. We call this type of dynamics Floquet oscillations since they arise from the periodic repetitions of Floquet quasienergy bands. So far very few works have addressed Bloch-type oscillations in the absence of an external lattice. One interesting prediction refers to Bloch oscillations of light, i.e., frequency oscillations of photons [20]. Further, Bloch-type oscillations were predicted theoretically for

*klaus.richter@physik.uni-regensburg.de interacting one-dimensional (1D) spinor gases [21] and recently observed in an atomic Bose liquid [22]. In these settings interactions lead to the dynamical formation of periodic structures, which can then yield Bloch oscillations. They do not, however, involve external drivings, and hence are of a different nature than the Floquet oscillations predicted here.

Specifically, we show that such periodic modes can emerge in spatially uniform systems governed by effective Dirac Hamiltonians, where the linear dispersion converts the energy periodicity of the Floquet spectrum into approximately $k$-periodic bands. Most notably, Floquet oscillations are a quantum phenomenon distinctly different from the classical oscillatory motion of a charge in an ac field. Instead of following the driving frequency, they exhibit a frequency inversely proportional to it. Moreover, Floquet oscillations offer a possibility to directly image the Floquet quasiband structure. Interestingly, they additionally show zitterbewegung features. We support our predictions by numerical calculations for two experimentally relevant prototypes of external driving, a periodic pulse sequence and circularly polarized radiation.

\section{GENERAL CONCEPT OF FLOQUET OSCILLATIONS}

Consider a system $H_{0}(\mathbf{k})$ (with momentum operator $\mathbf{k}$ ) subject to a time-periodic driving $V_{T}(t)$ with period $T$ and frequency $\omega=2 \pi / T$ described by the Hamiltonian

$$
H(\mathbf{k}, t)=H_{0}(\mathbf{k})+V_{T}(t)=H(\mathbf{k}, t+T) .
$$

Via Floquet theory [23-25] the problem is transformed to finding the quasienergy eigenvalues $\epsilon(\mathbf{k})$ of the Floquet Hamiltonian $\mathcal{H}_{F}(\mathbf{k})=H(\mathbf{k}, t)-i \hbar \frac{\partial}{\partial t}$. The quasienergies $\epsilon(\mathbf{k})$ extend to infinity in $\mathbf{k}$ space in the absence of a spatial lattice. However, they are periodic in quasienergy $\epsilon \in[-\hbar \omega / 2, \hbar \omega / 2]$ forming a sequence of Floquet replicas which are the ana$\log$ of the usual Bloch bands-the latter being periodic in quasimomentum $k \in[-\pi / a, \pi / a]$ due to spatial periodicity with lattice constant $a$. 


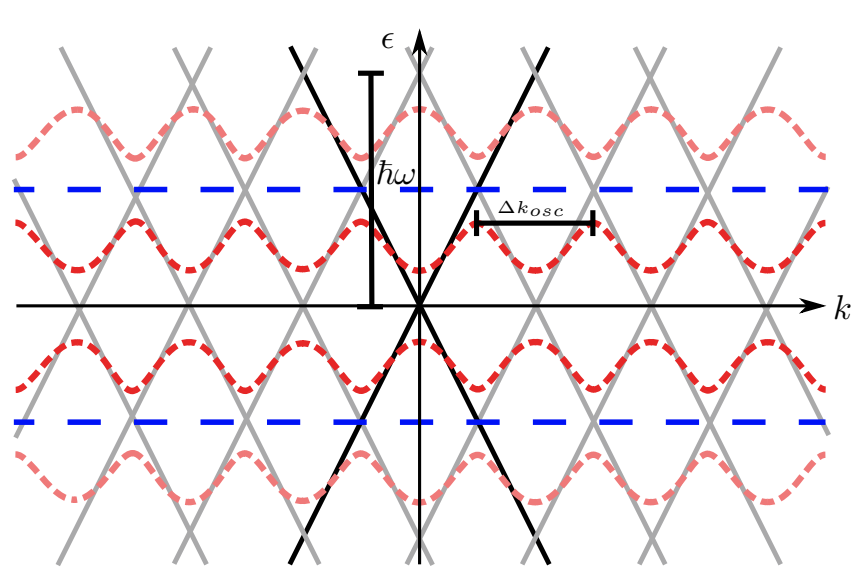

FIG. 1. Simplified scheme of the Floquet band structure $\epsilon_{ \pm}(k)$ of a Dirac system subject to a time-periodic potential. In Floquet theory the driving (at frequency $\omega$ ) introduces replicas of the original Dirac cone $E_{ \pm}= \pm \hbar v_{F} k$ (black), vertically shifted in energy by multiples of $\hbar \omega$ (thin gray). The blue dashed lines mark the first energy unit cell. For finite driving strength the bands hybridize and gaps open at the crossings, leading to distinct (approximately) $k$-periodic Floquet bands (red dashed) whose local maxima are $\Delta k_{\text {osc }}$ apart.

For the undriven system, we take an (effective) twodimensional Dirac Hamiltonian

$$
H_{0}(\mathbf{k})=\hbar v_{F} \mathbf{k} \cdot \boldsymbol{\sigma},
$$

with constant Fermi velocity $v_{F}$ and $\sigma=\left(\sigma_{x}, \sigma_{y}\right)$ the vector of Pauli matrices. The spectrum of $H_{0}(\mathbf{k})$ is composed of two energy branches $E_{ \pm}= \pm \hbar v_{F} k$ with $k=|\mathbf{k}|$. The Floquet bands $\epsilon_{ \pm}$that emerge when adding the time-periodic driving $V_{T}(t)$ to the system are sketched in Fig. 1. Due to the radial symmetry of the bands, we only show a cut along $k_{y}=0$. In the limit of infinitely small driving $V_{T}(t)$, the bare Dirac cone $E_{ \pm}$(black) is accompanied by a mesh of intersecting replicas (gray) that are shifted in energy by multiples of $\hbar \omega$. The blue dashed lines mark the corresponding first Brillouin zone (BZ) in energy. For finite $V_{T}(t)$ band gaps open at replica crossings and separated Floquet bands emerge (red dashed curves; see Appendix A for an introduction to the relevant aspects of Floquet theory). The band gaps sketched in Fig. 1 emerge since different replicas are coupled. Under the influence of the drift field $E$, a particle follows the band adiabatically along $k$ and successively absorbs/emits an increasing number $n$ of photons $\hbar \omega$ at each avoided crossing between two replicas of the (undriven) Dirac cone with a relative energy shift $n \hbar \omega$. Hence, the farther away from $k=0$, the more photons are needed to bridge the energy difference required for adiabatic dynamics associated with Floquet oscillations. As we will show below, time-periodic potentials $V_{T}$ with pronounced higher-order (in $\omega$ ) Fourier components will correspondingly open band gaps farther away from $k=0$ compared to, e.g., single frequency harmonic driving. Due to the underlying linear (Dirac) dispersion, the Floquet bands are approximately $k$ periodic, implying particularly pronounced Floquet oscillations with a well-defined frequency, in close analogy to Bloch oscillations [26].

To gain insight into the Floquet oscillation dynamics let us be definite and consider the time evolution of a wave packet
(WP) of Dirac electrons under the influence of an additional, constant electric field $\mathbf{E}=E \mathbf{e}_{x}$. Here, we consider the 1D motion along the field direction; generalizations to higher dimensions are straightforward. Due to the drift potential

$$
V(x)=-e E x, \quad e>0,
$$

the WP is accelerated and its initial wave vector $k_{i}$ changes linearly in time [18],

$$
k(t)=k_{i}-(e E / \hbar) t
$$

For ordinary Bloch bands, the $\mathrm{BZ}$ with period $\Delta k_{\mathrm{osc}}=2 \pi / a$ is traversed in time,

$$
T_{B}=2 \pi /(a|e E| \hbar) .
$$

While crossing the BZ, a charge carrier changes its velocity according to the change in slope of the $k$-periodic band structure, leading to a Bloch oscillation in $k(t)$ with frequency $\omega_{B}=2 \pi / T_{B}$.

For Floquet systems the velocity operator is given by $\hat{v}=$ $d \mathcal{H}_{F} / d k$ [18]. The diagonal terms of a WP's velocity read $\left\langle\hat{v}_{\alpha \alpha}\right\rangle=v_{\alpha \alpha}=d \epsilon_{\alpha} / d k$, with $\alpha= \pm$, and the corresponding position expectation value $\left\langle\hat{x}_{\alpha \alpha}\right\rangle=x_{\alpha \alpha}$ is obtained by time integration of $v_{\alpha \alpha}$. Using Eq. (4) this can be substituted by an integration over $k$ leading to

$$
x_{\alpha \alpha}(k(t))=\frac{\hbar}{e E}\left[\epsilon_{\alpha}(k(t))-\epsilon_{\alpha}\left(k_{i}\right)\right] .
$$

These diagonal contributions to $\langle\hat{x}\rangle(t)$ encode features of the Floquet band structure into the WP position. In particular, analogously to conventional Bloch oscillations, the WP is expected to perform oscillations for Floquet bands similar to the ones sketched in Fig. 1 (red dashed lines).

During one (Floquet) oscillation $k$ changes by the period $\Delta k_{\text {osc }}$ of the Floquet bands (Fig. 1). Hence, Eq. (4) implies the corresponding period $T_{F}=(\hbar /|e E|) \Delta k_{\mathrm{osc}}$. Due to the linear dispersion $E_{ \pm}(k)= \pm \hbar v_{F} k$, we have $\hbar v_{F} \Delta k_{\text {osc }}=\hbar \omega$ so that the Floquet oscillation period reads

$$
T_{F}=\frac{\hbar \omega}{v_{F}|e E|} .
$$

$T_{F}$ is proportional to the inverse period $1 / T=\omega /(2 \pi)$ of the driving in Eq. (1). While its analog, the Bloch period $T_{B}$ [Eq. (5)], is determined by the inverse (super)lattice constant $1 / a$, usually fixed in experiment, $T_{F}$ can be tuned through $\omega$ in a range such that $T_{F}>T$.

\section{RESULTS FOR REPRESENTATIVE DRIVING PROTOCOLS}

In the following we consider two representative types of driving well suited to generate Floquet oscillations: a periodic sequence of short pulses and a circularly polarized light field.

\section{A. Short pulse sequence}

For the first driving protocol we use

$$
V_{T}(t)=\sum_{l=1}^{\infty} \Theta(t-(l T-\Delta t)) \Theta(l T-t) M \sigma_{z}
$$



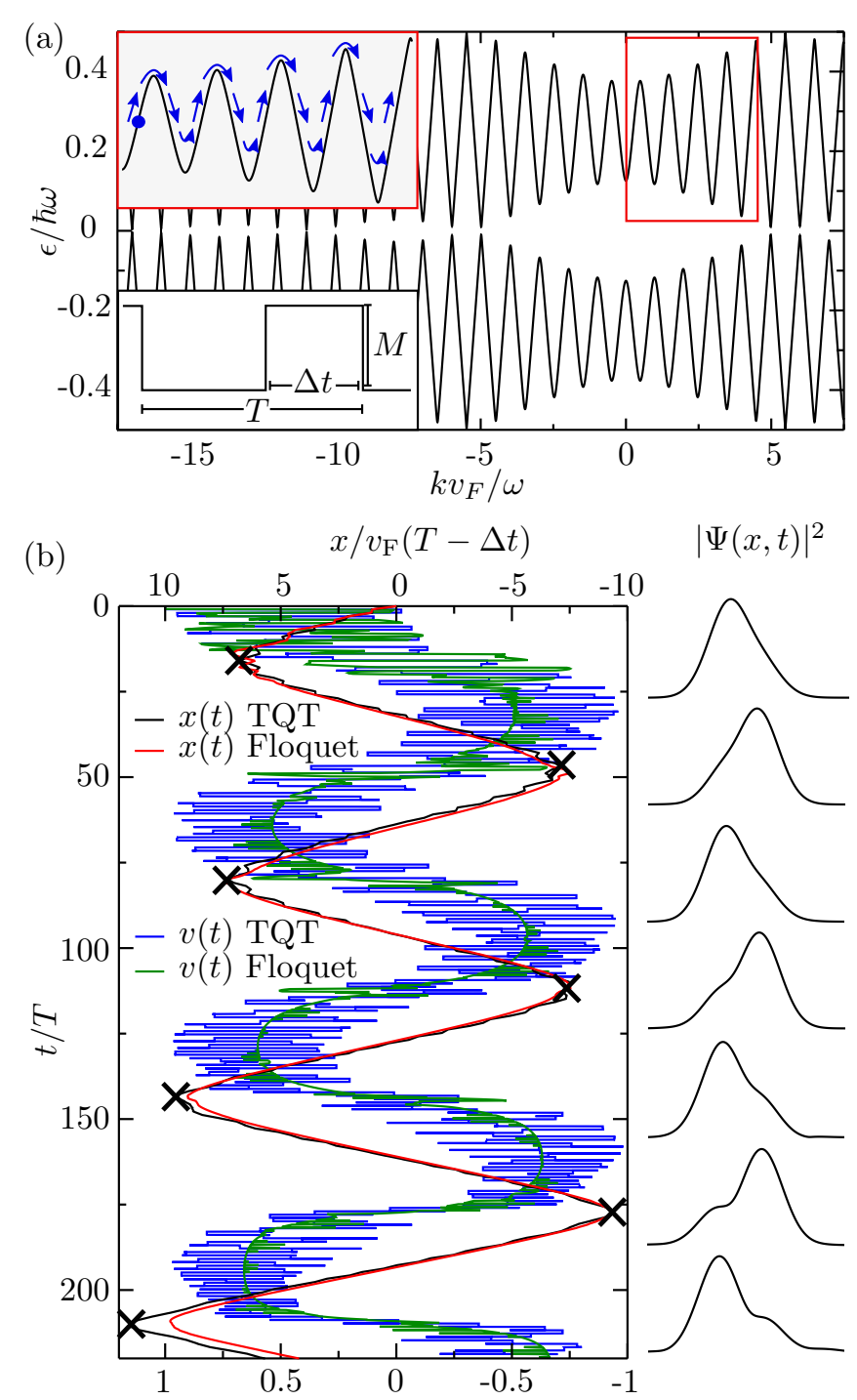

FIG. 2. Floquet oscillations in a driven, spatially homogeneous Dirac system. (a) Floquet bands from a periodically opened mass gap [see Eq. (8) and lower left inset for the pulse shape] with band gaps at the intersections of the unperturbed Dirac cones and their replicas (as sketched in Fig. 1). Two separated bands emerge in the energy window $\hbar \omega$. The motion of a WP (with initial position indicated by the blue dot) due to a static electric field [Eq. (3)] is sketched by the blue arrows in the zoomed-in region framed in red. (b) Left panel: Simulations of the WP position and velocity expectation values based on direct time evolution (TQT) and Floquet theory (see text for details). The numerical results confirm the Floquet oscillations mirroring the characteristic shape of the Floquet band structure. They also show zitterbewegung signatures. Right panel: Snapshots of the WP in position space taken at times marked as black crosses in the left panel.

in Eq. (1) (with Heaviside function $\Theta$ and Pauli matrix $\sigma_{z}$ ). This pulse train periodically couples the two branches of the Dirac Hamiltonian $H_{0}(\mathbf{k})$ [Eq. (2)] by opening a mass gap of strength $M$ and duration $\Delta t$ [see the lower inset in Fig. 2(a)]. The resulting Floquet spectrum can be tuned either by $M$ or by the ratio $\Delta t / T$. To be definite we choose the normalized pulse strength $M / \hbar \omega=4.4 / \pi$ and $\Delta t / T=0.09$.
The resulting Floquet band structure for this representative set of parameters is shown in Fig. 2(a). The driving opens a sequence of gaps around $\epsilon=0$ at the intersections of the original Dirac spectrum and its replicas. A detailed analysis of these Floquet bands and the $k$ dependence of the gaps is given in Appendix B.

A WP with initial quasienergy and wave number as marked by the blue dot in the zoomed-in area in Fig. 2(a) (red box, $\bar{k}_{i} v_{F} / \omega=0.25$ ) will undergo Floquet oscillations (blue arrows) when driven through the bands by a static electric field $E<0$ [Eq. (3)] such that $k$ increases in time [Eq. (4)]. Notably, since the Floquet band maxima and hence the bandwidth vary over $k$ space, the resulting Floquet oscillation is expected to change its amplitude but not its frequency. In our simulations we choose the field strength $E$ such that, according to Eq. (7), $T_{F} / T=2 \pi \hbar /\left(T^{2} v_{F}|e E|\right) \simeq 20.8 \pi$. We took initially Gaussian WPs of the form

$$
\tilde{\Psi}(k, 0)=\sqrt{\frac{1}{\sqrt{\pi} \Delta k}} \exp \left(-\frac{1}{2 \Delta k^{2}}\left(k-\bar{k}_{i}\right)^{2}\right)\left(\begin{array}{l}
1 \\
1
\end{array}\right),
$$

with width $\Delta k$ and initial central mode $\bar{k}_{i}$. We employ two complementary approaches to compute and analyze Floquet oscillations: Floquet theory and direct time integration of the full time-dependent effective Dirac equation including the E field.

To compute the WP velocity within Floquet theory, we start from Ehrenfest's theorem,

$$
v(t)=\langle\hat{v}(t)\rangle=\frac{i}{\hbar}\langle\Psi(t)|[H(t), \hat{x}]| \Psi(t)\rangle,
$$

where $[H(t), \hat{x}]=-i \hbar v_{F} \sigma_{x}$ for the Dirac case. $|\Psi(t)\rangle$ is obtained via the time-evolution operator of a Floquet system [27] that for a single $k$ mode reads

$$
U_{k}(t, 0)=\sum_{\alpha= \pm} \exp \left(-\frac{i}{\hbar} \epsilon_{\alpha}(k) t\right)\left|\phi_{\alpha, k}(t)\right\rangle\left\langle\phi_{\alpha, k}(0)\right| .
$$

Here, $\epsilon_{\alpha}$ are the Floquet quasienergies and $\left|\phi_{\alpha, k}(t)\right\rangle$ the corresponding eigenstates of $\mathcal{H}_{F}$, including replicas $n \hbar \omega$ [see Eq. (A3)], each consisting of two branches $\alpha= \pm$ from the linear dispersion. The additional electric field induces a linear change of $k$, which we account for by adjusting $k(t)$ according to Eq. (4). Applying $U_{k}(t, 0)$ to an initial (WP) state

$$
|\Psi(0)\rangle=\sum_{k_{i}} c_{\alpha, k_{i}}\left|\phi_{\alpha, k_{i}}(0)\right\rangle,
$$

where $\left|c_{\alpha, k_{i}}\right|^{2}=\left|\left\langle\phi_{\alpha, k_{i}}(0) \mid \Psi(0)\right\rangle\right|^{2}$ describes the initial occupation of branch $\alpha$, and plugging Eq. (11) into Ehrenfest's theorem gives

$$
\begin{aligned}
v(t)= & v_{F} \sum_{k_{i}} \sum_{\alpha, \beta= \pm} c_{\alpha, k_{i}}^{*} c_{\beta, k_{i}}\left\langle\phi_{\alpha, k(t)}(t)\left|\sigma_{x}\right| \phi_{\beta, k(t)}(t)\right\rangle \\
& \times \exp \left(-\frac{i}{\hbar}\left[\epsilon_{\beta}(k(t))-\epsilon_{\alpha}(k(t))\right] t\right) .
\end{aligned}
$$

Here, $k(t)$ is given by Eq. (4). The occupation $\left|c_{\alpha, k_{i}}\right|^{2}$ is time independent as long as different Floquet bands are far enough apart for Landau-Zener interband transitions [28-33] to be neglected. This is the case for the timescales $t \leqslant 200 T$ considered below. 
For the periodic pulse sequence [Eq. (8)] we numerically compute $v(t)$ by means of Eq. (13) and $x(t)=\int_{0}^{t} d t^{\prime} v\left(t^{\prime}\right)$. Due to the rectangular pulse shape we must include up to $n=$ 500 Floquet modes to achieve sufficient convergence. The results are shown in the left panel of Fig. 2(b) as red and green lines for $x(t)$ and $v(t)$, respectively. They indeed show distinct Floquet oscillations, as predicted, with period $T_{F} \simeq 20.5 \pi T$, matching the expected value $T_{F} \simeq 20.8 \pi T$ from Eq. (7). The off-diagonal velocity term $(\alpha \neq \beta)$ in Eq. (13) encodes the interference of states living in different Floquet bands, giving rise to an additional feature, zitterbewegung $[34,35]$ caused by the Floquet Dirac band structure (see Appendix C).

Our second, complementary method to compute Floquet oscillations is based on the WP propagation algorithm "time-dependent quantum transport" (TQT) [36] (see also Appendix D). The WP is discretized on a rectangular grid and the time-evolution operator for the full Hamiltonian including the drift field, $H(t)=H_{0}(\mathbf{k})+V_{T}(t)-E x$, is computed. Then the Lanczos method [37] is used to evaluate the action of the time-evolution operator on the WP and to compute $\Psi(x, t)$. The time-dependent position and velocity expectation values are then calculated through

$$
x(t)=\langle\hat{x}(t)\rangle=\int|\Psi(x, t)|^{2} x d x
$$

and

$$
v(t)=\langle\hat{v}(t)\rangle=\frac{d}{d t}\langle\hat{x}(t)\rangle
$$

The resulting TQT data are shown in the left panel of Fig. 2(b) as black and blue curves for $x(t)$ and $v(t)$, respectively. They approximately coincide with those computed within Floquet theory, also showing zitterbewegung on top of the Floquet oscillations. Moreover, the WP position $x(t)$ computed with TQT precisely reflects the characteristic features of the Floquet quasibands, shown in the red box in Fig. 2(a), namely, the increasing amplitude and the sharpening of the maxima and minima although TQT directly integrates the time-dependent Schrödinger equation without using Floquet formalism.

While the static electric drift field enters into the full Hamiltonian governing the numerically exact TQT time evolution, within the Floquet approach its effect is included via the acceleration theorem (4) into the time evolution, Eqs. (11) and (13). The latter approximation, together with residual numerical errors from the cutoff in the Floquet quantum number, could explain the slight deviations between Floquet and TQT data in the left panel of Fig. 2(b).

Finally, in the right panel of Fig. 2(b) we present snapshots of the absolute square $|\Psi(x, t)|^{2}$ taken at the turning points (marked as black crosses) of the red curve in the left panel. They show clear-cut Floquet oscillations of the full WP in configuration space generated for the setting of a periodic pulse sequence.

\section{B. Circularly polarized light}

The experimental realization of Floquet oscillations could be easier to achieve in an alternative setup, employing circularly polarized light as periodic driving. The associated vector potential A enters (linearly) the Dirac Hamiltonian (1) via the
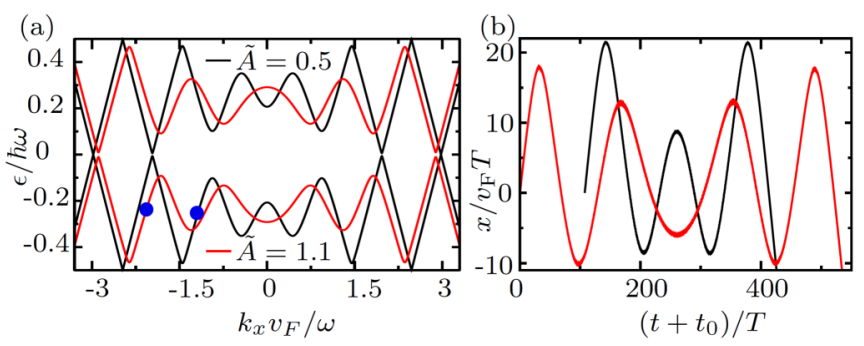

FIG. 3. (a) Floquet bands of a Dirac system illuminated with circularly polarized light with scaled amplitude $\tilde{A}=0.5$ (black curves) and 1.1 (red curves). (b) Floquet oscillations: Mean position of a WP driven through the bands shown in (a) starting with momenta $\bar{k}_{i}$ marked as blue dots in (a). The initial time $t_{0}$ for the black curve is shifted to $t_{0} / T=107.5$ to highlight the close connection to the Floquet band structure of (a). Additional oscillations due to the momentum change caused by the circulating electric field of the pulse are not resolved on this timescale.

minimal coupling

$$
V_{T}(t)=\mathbf{A}(t) \cdot \boldsymbol{\sigma}=A\left(\begin{array}{c}
\cos (\omega t) \\
\sin (\omega t)
\end{array}\right) \cdot \boldsymbol{\sigma} .
$$

The Floquet quasibands of graphene illuminated by circularly polarized light have already been studied extensively [38-41]. Recently, also transport [42] and topological [43-46] properties were investigated. Instead, here we focus on generating Floquet oscillations for realistic experimental parameters. To be closer to measurements, we explicitly treat the twodimensional (2D) case with an initial, radially symmetric Gaussian WP analogous to Eq. (9), with $k$ and $\bar{k}_{i}$ replaced by $\mathbf{k}$ and $\overline{\mathbf{k}}_{i}=\left(\bar{k}_{i}, 0\right)$. Using again TQT we simulate the WP dynamics (see Fig. 4) in the presence of the electric field $\mathbf{E}=E \mathbf{e}_{x}$, where $T_{F} / T=2 \pi \hbar /\left(T^{2} v_{F}|e E|\right) \simeq 140$.

We calculate the Floquet band structure and the WP dynamics for two different dimensionless driving strengths $\tilde{A}=$ $v_{F} e A /(\hbar \omega)$ to demonstrate the field amplitude influence on the Floquet oscillation frequency. Figure 3(a) shows the radially symmetric Floquet band structure along the direction of the electric field. For small enough driving strengths, the distance of local band minima is independent of $k$ (black). For larger driving amplitudes, multiple-photon processes significantly alter the formation of band gaps.

As displayed in Fig. 3(b), our TQT simulations of the position expectation values [Eq. (14)] of two WPs with scaled initial momenta $\bar{k}_{i} v_{F} / \omega=-2.07$ and -1.22 for $\tilde{A}=1.1$ and 0.5 , respectively [marked by blue dots in Fig. 3(a)], clearly show Floquet oscillations, nicely reflecting the shape of the underlying Floquet band structure as expected. Since the gaps between unperturbed Dirac cones open in a smaller $k$ range than for the periodic pulse train (8), there are less cycles of regular Floquet oscillations. At longer times Landau-Zener transitions to neighboring Floquet bands substantially alter the WP motion. Nevertheless, Fig. 3 shows Floquet oscillations with four full periods. In the famous experiments of Bloch oscillations in superlattices [5-7] and bulk semiconductors [13], their detection was possible even though only one to three periods could be achieved. Moreover, oscillations involving 

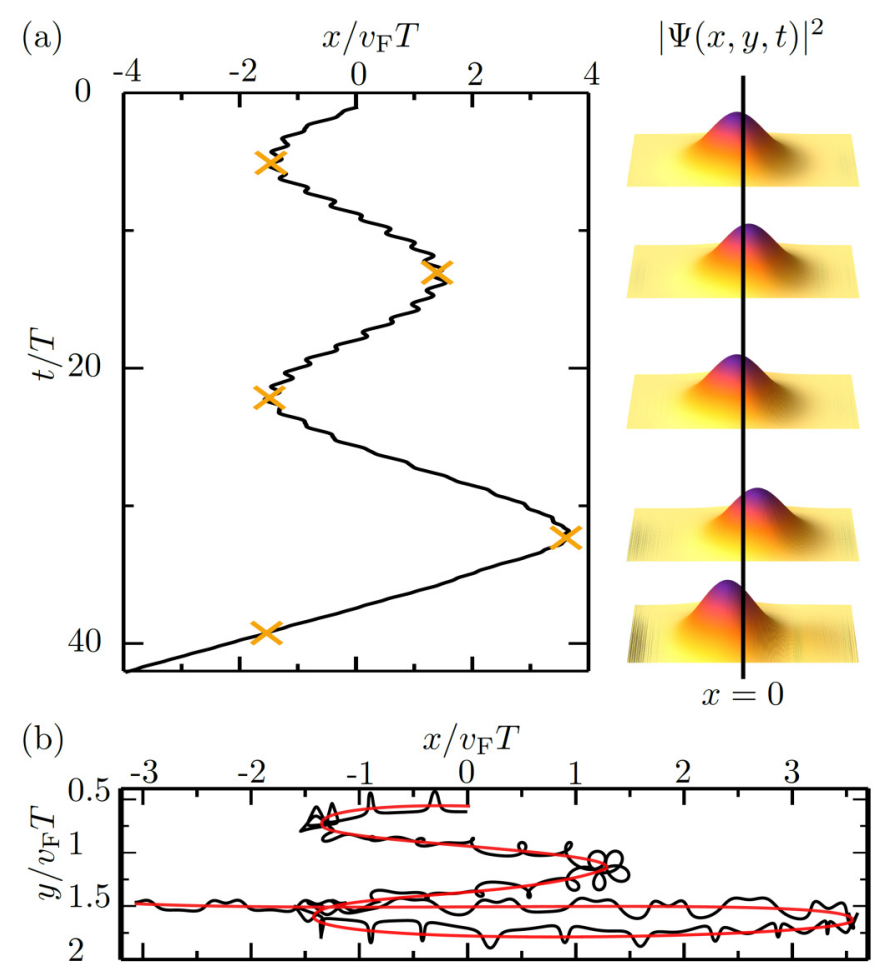

FIG. 4. (a) Simulation of the position expectation value of the WP in the direction of the electric field [Eq. (3)] [as shown in Fig. 3(b)] and its shape in position space. The orange crosses mark when the snapshots of the WP are taken. The tiny oscillations on top of the comparably slow Floquet oscillations are due to the momentum change caused by the circulating electric field of the pulse [Eq. (16)]. (b) Trajectory of the WP's center of mass $\langle\hat{\mathbf{x}}\rangle(t)$ displayed in (a) in the $x-y$ plane. The red curve has been included as a guide for the eye.

many periods exist for the case of periodic kicking which can be experimentally realized through laser pulse trains.

In analogy to Fig. 2(b), Fig. 4(a) shows the position expectation value [Eq. (14)] along the electric field [Eq. (3)] and the 2D shape of the WP. Here, we choose $\bar{k}_{i} v_{F} / \omega=$ -0.64 and $T_{F} / T=2 \pi \hbar /\left(T^{2} v_{F}|e E|\right) \simeq 14$ without shifting the initial time $t_{0}$ and including orange crosses to mark when the snapshots of the WP are taken. The snapshots displayed on the right show the absolute square of the WP in 2D space and the oscillation of its center of mass around $x=0$. Note that, according to Eq. (7), $T_{F}$ is inversely proportional to $E$, therefore in Fig. 4 the timescale of the Floquet oscillations is a factor of 10 smaller than in Fig. 3. Here, the oscillations induced by the circulating electric field [Eq. (16)] are resolved on top of the slower Floquet oscillations. In Fig. 4(b), we plot the trajectory of the WP's center of mass in the $x-y$ plane. The red curve is meant to serve as a guide for the eye. In the trajectory, one can recognize the Floquet oscillations but also some additional features. The smaller oscillations have the same origin as the tiny oscillations on top of the comparably slow Floquet oscillations in Fig. 4(a): They arise from the momentum change due to the circulating electric field of the pulse [Eq. (16)] and are modulated by zitterbewegung. The drift of the WP in the $y$ direction, however, has a less intuitive explanation. We assume that it is caused by the anomalous velocity, i.e., the Berry curvature of the Floquet bands, since it is orthogonal to the electric driving field. This assumption will be tested in future studies.

\section{Including trigonal warping and introducing experimental parameters}

Finally, we want to give a few more details on the experimental realizability of Floquet oscillations in graphene. First, we numerically verify that when choosing the correct energy scales, trigonal warping effects do not play a role. Therefore we extend our Hamiltonian $H_{0}$ (k) [Eq. (2)] to

$$
H(\mathbf{k})=\hbar v_{F} \mathbf{k} \cdot \boldsymbol{\sigma}-\mu\left[\left(k_{y}^{2}-k_{x}^{2}\right) \sigma_{x}+2 k_{x} k_{y} \sigma_{y}\right],
$$

with $v_{F}=10^{6} \mathrm{~m} / \mathrm{s}$ and $\mu=3 a^{2} t / 8$, where $a=1.42 \AA$ is the nearest-neighbor distance and $t=2.7 \mathrm{eV}$ the nearestneighbor hopping strength of graphene [47]. We set the light amplitude $A=45 \mathrm{nV} \mathrm{s} / \mathrm{m}$ and the frequency $\omega / 2 \pi=$ $10 \mathrm{THz}$. We simulate the wave packet motion for $\bar{k}_{i}=$ $0.0131 / \AA$. The described parameters are equivalent to the unitless values chosen for Fig. 3. In Fig. 5 we compare the Floquet oscillations obtained with and without the trigonal warping term and find no qualitative differences. However, for experimental realization in graphene the transport relaxation time $\tau$ has to be higher than the period $T_{F}$ of the Floquet oscillations [Eq. (7)], typically $\tau=1-20$ ps [48,49]. As $T_{F} \simeq 13.5 \mathrm{ps}$ for $E=30 \mathrm{~V} / \mathrm{cm}$ [Fig. 5(a)], good quality samples would already allow for the observation of one period. If we increase the electric field by a factor of 10 [Fig. 5(b)], $T_{F} \simeq 1.3$ ps. Then, all four Floquet oscillation cycles could be detected. Note that for the higher electric field the Floquet oscillations become slightly altered. The additional oscillations are caused by zitterbewegung and the momentum change induced by the circularly polarized light field and are resolved here since the Floquet oscillations occur on the timescale of picoseconds.

\section{CONCLUSIONS}

The above analysis and simulations constitute a proof of principle for generating Floquet oscillations in systems with an effective Dirac dispersion. Concerning possible experimental realizations, graphene [50], topological insulators [51,52], and cold atoms in artificial honeycomb lattices [53] appear suitable [54,55]. The latter have the advantages that one can additionally tune $v_{F}$ entering the Floquet frequency and that relaxation through disorder or interaction effects can be avoided. Other effective Dirac systems, e.g., for plasmons [56] or polaritons [57], could also be considered. In the following, we will quantitatively focus on realizations for charge carriers in real monolayer graphene.

The radiation frequency $\omega$ must be chosen such that several Floquet BZs lie in the energy range governed by the linear dispersion. In graphene, a frequency $v=2 \pi \omega$ of $1-10 \mathrm{THz}$ is small enough to accommodate roughly 50-5 Floquet replicas over the energy range of $400 \mathrm{meV}$, for which the linear Dirac cone is a very good approximation. Additionally, $\omega>\omega_{F} \gg 2 \pi / \tau$ is required, where $\tau$ denotes a typical relaxation time of charge carriers. To realize the oscillations shown in Fig. 5(b) with $\tilde{A}=1.1$ at a radiation frequency of 


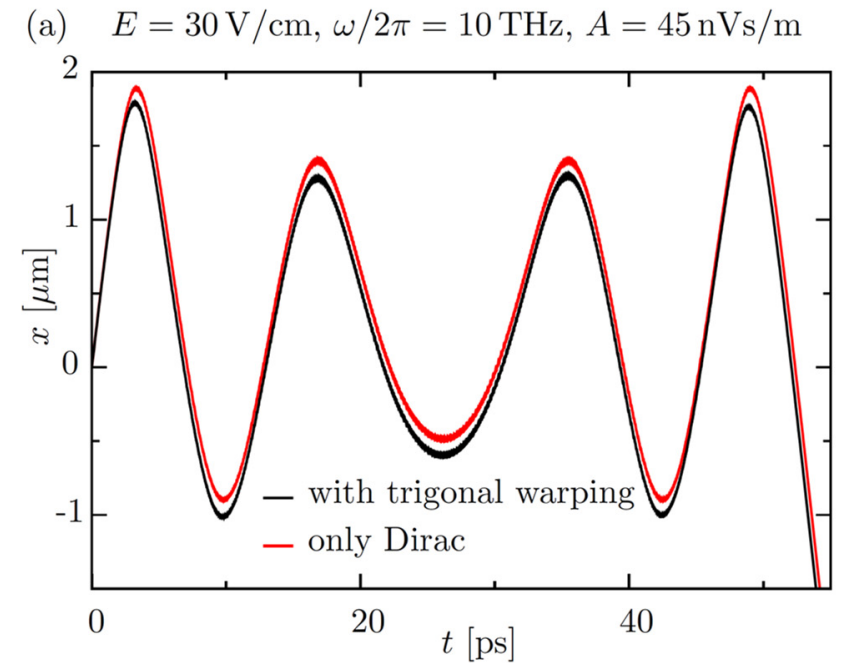

(b) $E=300 \mathrm{~V} / \mathrm{cm}, \omega / 2 \pi=10 \mathrm{THz}, A=45 \mathrm{nVs} / \mathrm{m}$

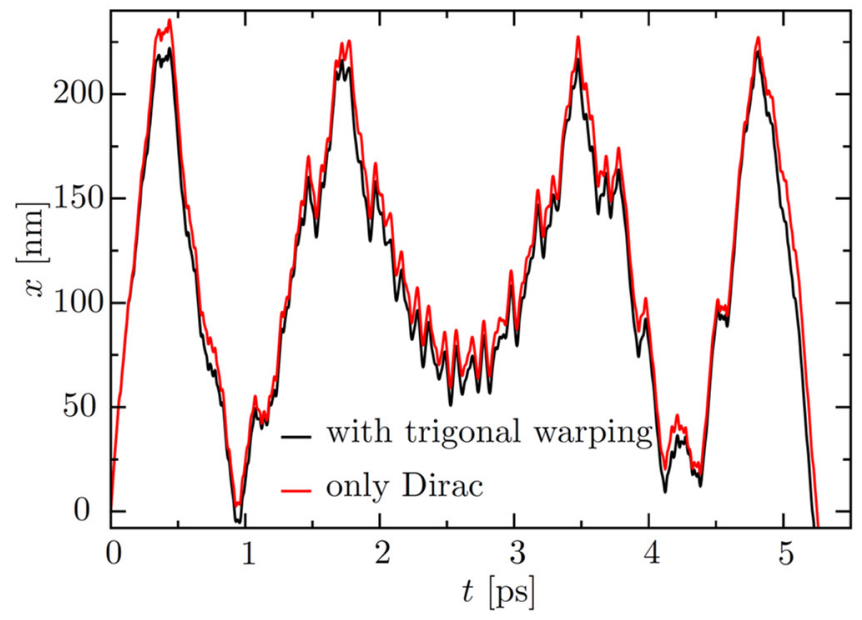

FIG. 5. Floquet oscillations in graphene with a driving frequency $\omega / 2 \pi=10 \mathrm{THz}$ and laser amplitude $A=45 \mathrm{nV} \mathrm{s} / \mathrm{m}$. (a) When a static electric field $E=30 \mathrm{~V} / \mathrm{cm}$ is applied, clear Floquet oscillations of period $T_{F} \simeq 13.5 \mathrm{ps}$ are visible. (b) Increasing the electric field $E$ by a factor of 10 reduces the Floquet period to $T_{F} \simeq$ $1.3 \mathrm{ps}$. Because of the smaller timescale, the oscillations due to the momentum change induced by the circularly polarized light $(T=$ $0.1 \mathrm{ps}$ ) are now resolved. Their features are additionally altered by zitterbewegung. For both cases (a) and (b) including trigonal warping in the calculations does not lead to qualitative changes of the Floquet oscillations.

$\omega / 2 \pi=10 \mathrm{THz}$, a moderate intensity (avoiding sample heating) of $I=\frac{c \epsilon_{0}}{2} A^{2} \omega^{2} \simeq 1 \mathrm{MW} / \mathrm{cm}^{2}$ is needed. Here, $c$ is the speed of light and $\epsilon_{0}$ the vacuum permittivity. Then an electric field $E \simeq 0.3 \mathrm{kV} / \mathrm{cm}$ is sufficient to generate Floquet oscillations of frequency $\omega_{F} / 2 \pi \gtrsim 1 \mathrm{THz}$. Hence $\omega_{F} \gtrsim$ $2 \pi / \tau$ for typical inverse transport relaxation times $1 / \tau=$ $0.05-1 \mathrm{THz}$ of clean hexagonal boron nitride-encapsulated graphene $[48,49]$. Thus, Floquet oscillations could in principle be observed, opening an alternative way to generate $\mathrm{THz}$ radiation $[58,59]$.

To conclude, we showed that free particles in a static electric drift field and obeying a linear Dirac-type dispersion can perform spatially periodic motion, Floquet oscillations, when subject to time-periodic driving. The Floquet time lattice takes on the role of the spatial lattice required for conventional Bloch oscillations. Such Floquet oscillations feature zitterbewegung and characteristic amplitude modulations that could provide a tool to experimentally map the Floquet quasibands. A closer consideration of Landau-Zener transitions between different Floquet bands and the question of how the topology of Floquet bands [14] is reflected in corresponding Floquet oscillations opens interesting perspectives for future research.

\section{ACKNOWLEDGMENTS}

We thank Simon Maier for support with calculating Floquet band structures at an early stage of this work and Sergey Ganichev for helpful discussions and careful reading of the manuscript. We further thank an anonymous referee for suggesting to interpret Floquet oscillations in terms of multiple photon exchange. We acknowledge funding by the Deutsche Forschungsgemeinschaft (DFG, German Research Foundation) Project-ID 314695032-SFB 1277 (project A07).

\section{APPENDIX A: BASIC RELATIONS IN FLOQUET THEORY}

In the following we describe how to obtain the Floquet band structure for a finite driving strength $V_{T}(t)$ without an electric field, which is later included by a shift of $k$ when computing Floquet oscillations. Generally, the Floquet operator $\mathcal{H}_{F}$ is given by

$$
\mathcal{H}_{F}=H_{0}(\mathbf{k})+V_{T}(t)-i \hbar \partial_{t},
$$

where $H_{0}(\mathbf{k})$ is the Hamiltonian of the time-independent system. Since the eigenstates of $\mathcal{H}_{F}, \Phi_{\alpha}(\mathbf{k}, t)$, are periodic in time, they can be expanded in a Fourier series,

$$
\Phi_{\alpha}(\mathbf{k}, t)=\sum_{n=-\infty}^{\infty} \mathbf{u}_{\alpha}(\mathbf{k}, n) e^{i n \omega t} .
$$

The dimension of the coefficients $\mathbf{u}_{\alpha}(\mathbf{k}, n)$ is equal to the number of branches $\alpha$ of the time-independent Hamiltonian $H_{0}(\mathbf{k})$, i.e., two in our case. In order to find these coefficients and the corresponding eigenenergies $\epsilon_{\alpha}$, the Floquet equation (A1) is multiplied by $e^{-i m \omega t}, m \in \mathbb{Z}$, and averaged over one period $T$ to end up with $[60,61]$

$$
\sum_{n=-\infty}^{\infty} \underbrace{\left(\mathcal{H}_{0 F, m n}(\mathbf{k})+V_{F, m n}\right)}_{\mathcal{H}_{F, m n}(\mathbf{k})} \mathbf{u}_{\alpha}(\mathbf{k}, n)=\epsilon_{\alpha}(\mathbf{k}) \mathbf{u}_{\alpha}(\mathbf{k}, m) .
$$

Here, the contributions from $V_{T}(t)$ are denoted by $V_{F, m n}$ and the contributions from $H_{0}(\mathbf{k})$ and $-i \hbar \partial_{t}$ by $\mathcal{H}_{0 F, m n}(\mathbf{k})$. Note that the resulting eigenvalue problem is time independent and all the dynamics of a WP in the system are incorporated in the Floquet basis states [18]. To account for this when projecting a WP from the time-dependent basis to the Floquet basis, the relation $\left\langle k_{o}(t)\right\rangle_{t}=k_{\text {Floquet }}$ is used, where $\left\langle k_{o}(t)\right\rangle_{t}=$ $\frac{1}{T} \int_{0}^{T} k_{o}(t) d t$. The time dependence of $k_{o}(t)$ is introduced by the time-periodic driving $V_{T}(t)$. Hereafter, we refer to $k_{\text {Floquet }}$ when talking of wave numbers and suppress its subscript to simplify the notation. The Floquet Hamiltonian without 
coupling,

$$
\mathcal{H}_{0 F, m n}(\mathbf{k})=\left(\hbar v_{F} \mathbf{k} \cdot \boldsymbol{\sigma}+m \hbar \omega\right) \delta_{m n},
$$

is diagonal and describes the Dirac band structure shown in Fig. 1. The driving Hamiltonian,

$$
V_{F, m n}=\frac{1}{T} \int_{0}^{T} d t V_{T}(t) e^{i(n-m) \omega t},
$$

on the other hand, couples different Floquet modes $\mathbf{u}_{\alpha}(\mathbf{k}, n)$ and thus can lead to the opening of band gaps in the originally linear spectrum (see Fig. 1). For the numerical evaluation, the resulting infinite matrix $\mathcal{H}_{F}$ has to be truncated at a finite value $\pm n$ that corresponds to the number of Floquet replicas taken into account. When performing numerical calculations, one has to make sure that the results are converged for the chosen value of $n$.

\section{APPENDIX B: FLOQUET QUASIBAND STRUCTURE OF A DIRAC SYSTEM WITH A PERIODICALLY OPENED MASS GAP}

Here, we give a more detailed analysis of the Floquet band structure of a Dirac system with a periodically opened mass gap. The potential describing this time-dependent pulsing is given in Eq. (8). For the mass gap, we find with Eq. (A5),

$$
V_{F, m n}=\frac{M}{2 \pi i(n-m)}\left(1-e^{-2 \pi i(n-m) \frac{\Delta t}{T}}\right) \sigma_{z} .
$$

Qualitatively, the two timescales $\Delta t$ and $T$ involved are reflected in $k$ space. While the longer scale $T$ makes for the high-frequency oscillations of the Floquet bands due to the replicas, the smaller timescale $\Delta t$ is responsible for the slow modulation, i.e., the different gap sizes as a function of $k$ shown in Fig. 6.

On a more quantitative level, the dependence of the Floquet bands on the pulse amplitude $M$ and pulse duration $\Delta t$ can be best understood when studying the influence of one pulse on a WP in the static Dirac cone. This has been done extensively in Ref. [62] and will only be summarized here. Let us consider a WP initially occupying states in the upper cone. The opening and closing of the gap causes a redistribution of the WP to both cones, leading to a new superposition state. The amplitude of the part now occupying the other cone-in our example the lower one - can easily be calculated analytically [62],

$$
A(k)=-\frac{i}{\sqrt{1+\eta^{2}}} \sin \left(\mu \sqrt{1+\eta^{2}}\right),
$$

where $\eta=E_{k, \pm} / M$ and $\mu=M \Delta t / \hbar$. This transition amplitude only depends on the initial energy $E_{k, \pm}$ of the state and the pulse parameters. A numerical comparison of the corresponding transition probability $P(k)=|A(k)|^{2}$ and the Floquet band structure reveals that the gaps that open at the intersections of the repetitions of the original cone are directly related to the transition probability at that $k$ value: The larger $P(k)$, the larger is the band gap. The reason for this dependence can be motivated in the following way. $P(k)$ describes for a single pulse the proportion of the WP that is transferred to the other band, i.e., how much a single pulse couples the upper and lower band of the Dirac cone. On the
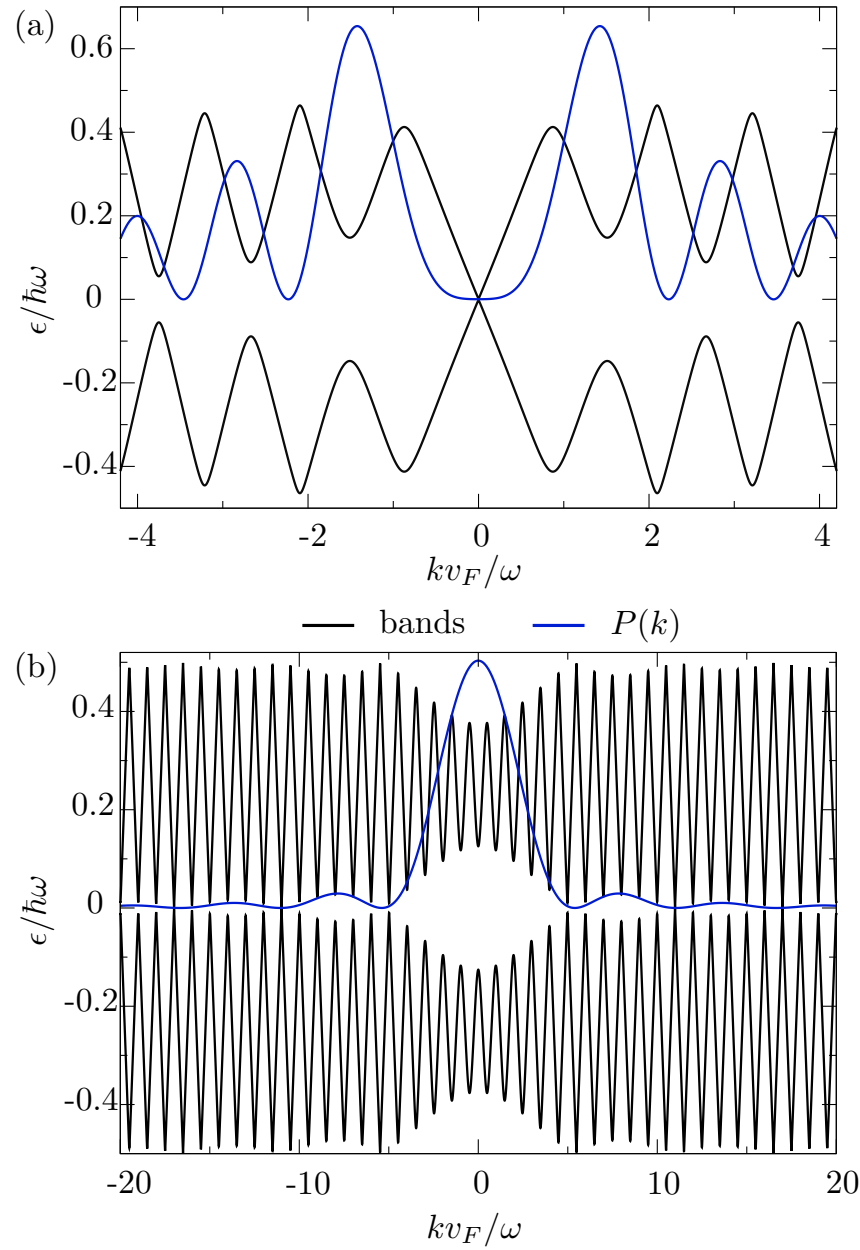

FIG. 6. Floquet band structure (black curves) and corresponding transition probability $P(k)$ (blue) for (a) $\mu=6.3, \Delta t / T=0.5$ and (b) $\mu=0.4, \Delta t / T=0.09$. The bands shown in Fig. 2(a) of the main paper are a zoom into those depicted in (b). For both parameter sets one can easily see that the gap in the Floquet bands closes whenever $P(k)$ goes to zero.

other hand, the band gap of the Floquet bands is due to this coupling of (initially) linear band replicas. Therefore, it is not surprising that $P(k)$ and the band gap width are directly related.

We show this for two exemplary band structures in Fig. 6. In Fig. 6(a) we set $\mu=6.3$ and $\Delta t / T=0.5$. Since $P(k)=0$ around $k v_{F} / \omega=0$ the original Dirac cone is preserved. For larger $k$, the band gaps open. The resulting complex band structure is a perfect example of how nicely the Floquet band structure can be tuned based on the transition probability $P(k)$. The band structure shown in Fig. 6(b) is the same as the one shown in Fig. 2(a) of the main paper but for a larger range of $k$ values. There, a wide area around $k v_{F} / \omega=0$ is gapped. For an appropriate choice of parameters [as in Fig. 6(b)] a large $k$ window, in which Landau-Zener transitions are suppressed, can be chosen to support Floquet oscillations. As a rule of thumb, the smaller $\Delta t / T$, the more the band gaps open and thus allow for more periods of Floquet oscillations before Landau-Zener transitions diminish them. 


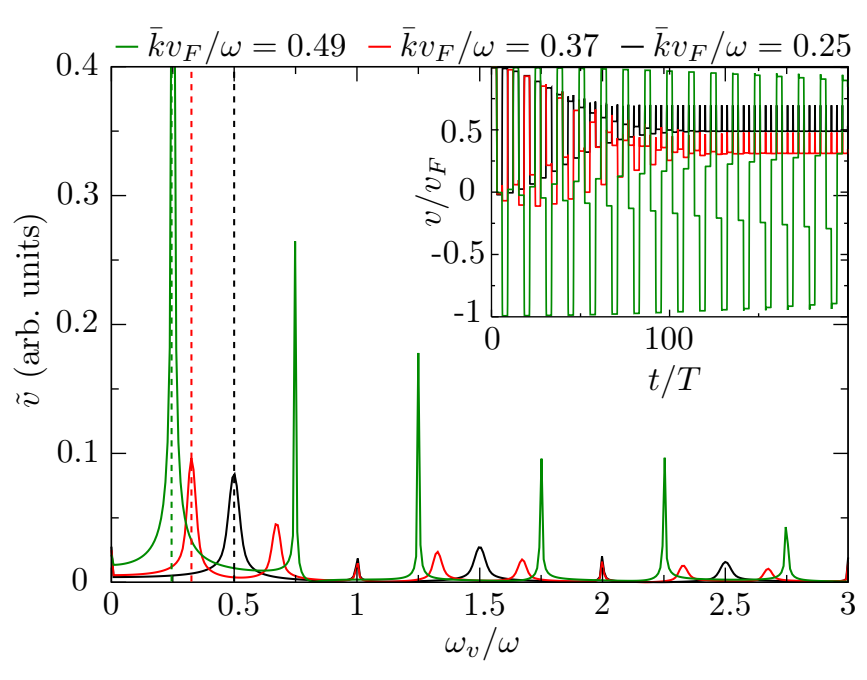

FIG. 7. Frequency analysis by a Fourier transform of the velocity $\tilde{v}\left(\omega_{v}\right)$ of WPs with different $\bar{k}$. Since no electric field is applied, $k$ is constant. The dashed lines mark $\omega_{\mathrm{ZB}}$ as expected from Eq. $(\mathrm{C} 1)$ which match perfectly the numerically obtained peaks. The inset shows the corresponding time dependency of the velocities. For the Fourier analysis, the respective average velocities have been subtracted to avoid a zero-frequency peak.

\section{APPENDIX C: ZITTERBEWEGUNG IN A DIRAC SYSTEM WITH A PERIODICALLY OPENED MASS GAP}

Zitterbewegung (ZB) was originally predicted by Schrödinger for the Dirac equation [34] but an analog is also visible in multiband systems [35,63-67]. The reason is the interference of particle and antiparticle contributions in a WP, or respectively, the contributions of different bands, in cases when the velocity operator does not commute with the Hamiltonian. The corresponding term is the off-diagonal term of the expectation value of the velocity operator and the frequency is given by the difference of the energies. In our case, we also have an effective two-band model due to the Floquet bands with an off-diagonal term of the velocity as seen in Eq. (13). The frequency of the corresponding "Floquet zitterbewegung" is given by the energy gap between the two Floquet quasienergies,

$$
\omega_{\mathrm{ZB}}=\left[\epsilon_{\beta}(k)-\epsilon_{\alpha}(k)\right] / \hbar .
$$

In Fig. 7 we show the Fourier transform $\tilde{v}\left(\omega_{v}\right)$ of the velocity of a WP starting at different $k$ values in the Floquet band structure to analyze the frequency spectrum. We denote the variable of the Fourier transform of the velocity by $\omega_{v}$. For these calculations no static electric field was applied, such that $k$ and therefore $\omega_{\mathrm{ZB}}$ stay constant in time. Before performing the Fourier transform to investigate the frequency of the oscillations, the mean velocity value was subtracted of the corresponding data to avoid a peak at $\omega_{v}=0$.

The dashed lines mark $\omega_{\mathrm{ZB}}$ as calculated by Eq. (C1). Their good agreement with the spectrum confirms that the offdiagonal velocity in the Floquet picture describes ZB caused by the interference of states occupying different Floquet bands. Since the velocity has a rectangular shape, the peaks are repeated at higher harmonics. This rectangular shape can be explained in our example by the fact that the velocity can only change while the mass gap is open, which means that the harmonic oscillation of the ZB is effectively sampled with the driving frequency $\omega$.

\section{APPENDIX D: THE C++ LIBRARY "TIME-DEPENDENT QUANTUM TRANSPORT" (TQT)}

To propagate a quantum state $|\psi\rangle$, one has to solve the time-dependent Schrödinger equation,

$$
i \hbar \frac{\partial}{\partial t}|\psi\rangle=\hat{H}|\psi\rangle,
$$

with the Hamilton operator $\hat{H}$, which depends in general on time. Formally, it can be solved using the time-evolution operator

$$
U\left(t, t_{0}\right)=\mathcal{T} \exp \left(-\frac{i}{\hbar} \int_{t_{0}}^{t} \hat{H}\left(t^{\prime}\right) d t^{\prime}\right),
$$

which is unitary and fulfills

$$
U\left(t, t_{0}\right)=U\left(t, t^{\prime}\right) U\left(t^{\prime}, t_{0}\right),
$$

where $t_{0}$ is the initial time, $t$ is some arbitrary later time, and $t^{\prime}$ is a time in between. The time evolution of a state then yields

$$
|\psi(t)\rangle=U\left(t, t_{0}\right)\left|\psi\left(t_{0}\right)\right\rangle .
$$

Moreover, for time-independent Hamiltonians, the timeevolution operator simplifies to

$$
U\left(t_{0}, t\right)=\exp \left(-i \frac{\hat{H}}{\hbar}\left(t-t_{0}\right)\right) .
$$

On the other hand, any function can be approximated by stepwise constant functions- the smaller the steps, the better is the approximation. Thus, the time-ordered exponential of Eq. (D2) can be estimated by

$$
U\left(t_{0}, t_{0}+N \delta t\right) \approx \prod_{j=0}^{N-1} \exp \left(-i \frac{\hat{H}\left(t_{0}+j \delta t\right)}{\hbar} \delta t\right),
$$

where the Hamiltonian is made stepwise constant for the time duration $\delta t$. The advantage is that instead of the time-ordered product of Eq. (D2), a rather easy multiplication can be performed. Of course, one has to make sure that the time step $\delta t$ is small enough, such that the numerical result is converged.

Using the time-evolution operator, we shifted the problem of solving the differential equation in Eq. (D1), to having the Hamilton operator in an exponential, which is defined by its (infinite) series expansion. The publicly available $\mathrm{C}++$ library "time-dependent quantum transport" (TQT) by Krueckl [36] takes care of this expansion as efficiently as possible for $1 \mathrm{D}$ or $2 \mathrm{D}$ systems. The expanded time-evolution operator acts on a numerically defined initial state in real space. Since a sufficiently smooth function can be approximated by its values at discrete points, the space is discretized by a grid, in 1D with $N_{x}$ and in 2D with $N_{x} \times N_{y}$ points. Thus, the wave function becomes a complex valued $N_{x}$-component vector or $\left(N_{x} \times N_{y}\right)$ matrix, respectively. For this work we usually take $N_{x}=8192$ for $1 \mathrm{D}$ and $N_{x} \times N_{y}=8192 \times 256$ for $2 \mathrm{D}$ systems.

The Hamiltonian can be either given as a tight-binding Hamiltonian or as a mixed position and momentum space 
representation, i.e., a function of both the position and momentum operator, the latter being the Hamiltonian used in most cases for analytical calculations. In the mixed representation, instead of using the spatial derivative, the momentum operator acts in momentum space, i.e., the wave function is transformed by a fast Fourier transform, then the momentum operator acts as a factor, and finally the inverse fast Fourier transformation is applied to get back to position space. The reason for using the Fourier transformation instead of the derivative is the numerical instability of the latter. Since the momentum operator acts several times (in higher orders $k_{i}^{n}$ ) in each small time step, the errors add up quickly. In this paper, only the mixed representation of the position and momentum operator is used.

Due to the explicitly time-dependent Hamiltonian in our problem, a Lanczos method is used to expand the timeevolution operator $[68,69]$ instead of a Chebyshev expansion $[70,71]$. The difference here is that instead of expanding in a fixed set of polynomials, the time-evolution operator is expanded in terms of the wave function $\psi$ itself and powers of the Hamiltonian acting on the wave function $\hat{H}^{n} \psi$. The thereby spanned subspace is an $N$-dimensional Krylov subspace $\mathcal{K}=\operatorname{span}\left\{\psi, \hat{H} \psi, \ldots, \hat{H}^{N-1} \psi\right\}$, which is orthonormalized to get the basis vectors $u_{n}$ by a Gram-Schmidt procedure during the recursive creation for better numerical stability,

$$
\begin{gathered}
u_{0}=\frac{\psi\left(t_{0}\right)}{\left|\psi\left(t_{0}\right)\right|}, \\
u_{1}=\frac{\hat{H} u_{0}-\alpha_{0} u_{0}}{\beta_{0}}, \\
u_{n+1}=\frac{\hat{H} u_{n}-\alpha_{n} u_{n}-\beta_{n-1} u_{n-1}}{\beta_{n}},
\end{gathered}
$$

[1] F. Bloch, Z. Phys. 52, 555 (1929).

[2] C. Zener, Proc. R. Soc. London, Ser. A 145, 523 (1934).

[3] For a review, see A. Wacker, Phys. Rep. 357, 1 (2002).

[4] For an early proposal to employ superlattices, see L. Keldysh, Sov. Phys. - Solid State 4, 1658 (1963).

[5] J. Feldmann, K. Leo, J. Shah, D. A. B. Miller, J. E. Cunningham, T. Meier, G. von Plessen, A. Schulze, P. Thomas, and S. Schmitt-Rink, Phys. Rev. B 46, 7252(R) (1992).

[6] C. Waschke, H. G. Roskos, R. Schwedler, K. Leo, H. Kurz, and K. Köhler, Phys. Rev. Lett. 70, 3319 (1993).

[7] A. A. Ignatov, E. Schomburg, K. F. Renk, W. Schatz, J. F. Palmier, and F. Mollot, Ann. Phys. 506, 137 (1994).

[8] M. Ben Dahan, E. Peik, J. Reichel, Y. Castin, and C. Salomon, Phys. Rev. Lett. 76, 4508 (1996).

[9] S. R. Wilkinson, C. F. Bharucha, K. W. Madison, Q. Niu, and M. G. Raizen, Phys. Rev. Lett. 76, 4512 (1996).

[10] T. Pertsch, P. Dannberg, W. Elflein, A. Bräuer, and F. Lederer, Phys. Rev. Lett. 83, 4752 (1999).

[11] R. Morandotti, U. Peschel, J. S. Aitchison, H. S. Eisenberg, and Y. Silberberg, Phys. Rev. Lett. 83, 4756 (1999).

[12] H. Sanchis-Alepuz, Y. A. Kosevich, and J. Sánchez-Dehesa, Phys. Rev. Lett. 98, 134301 (2007). with the overlaps $\alpha_{n}=\left\langle u_{n}|\hat{H}| u_{n}\right\rangle$ and $\beta_{n-1}=\left\langle u_{n-1}|\hat{H}| u_{n}\right\rangle$. Note that $u_{n}$ is a linear combination of powers of $\hat{H}$ acting on $\psi$, with highest order $n$.

The truncated Hamiltonian in this subspace becomes tridiagonal,

$$
H_{\mathcal{K}}=\left(\begin{array}{ccccc}
\alpha_{0} & \beta_{0} & 0 & \cdots & 0 \\
\beta_{0} & \alpha_{1} & \beta_{1} & & 0 \\
0 & \beta_{1} & \alpha_{2} & & 0 \\
\vdots & & & \ddots & \beta_{N-2} \\
0 & \cdots & 0 & \beta_{N-2} & \alpha_{N-1}
\end{array}\right),
$$

which can be diagonalized by conventional algorithms and enables the calculation of approximate eigenvalues of the operator $\hat{H}$ [37]. With the matrix of eigenvectors $\mathbf{T}$ and eigenvalues $\mathbf{E}$ of the Hamiltonian in the reduced Krylov space $H_{\mathcal{K}}$, the time evolution of one small time step is given by

$$
\psi(t+\delta t)=\sum_{n=0}^{N-1}\left[\mathbf{T}^{t} \exp \left(-\frac{i}{\hbar} \mathbf{E} \delta t\right) \mathbf{T} \psi_{\mathcal{K}}(t)\right]_{n} \cdot u_{n}
$$

The expansion in the Krylov subspace is faster than a Taylor expansion [72], and for the Krylov space, a dimension $N$ in the range 10-40 is usually enough. It turned out that for the calculations in this paper, the dimension of the Krylov space of $N=15$ is sufficient.

With the thus obtained time-dependent state on our discrete timeline, an arbitrary (observable) quantity such as the position expectation value can be obtained as a function of the time for the propagation, which yields in our case the Floquet oscillations.
[13] O. Schubert, M. Hohenleutner, F. Langer, B. Urbanek, C. Lange, U. Huttner, D. Golde, T. Meier, M. Kira, S. W. Koch, and R. Huber, Nat. Photonics 8, 119 (2014).

[14] N. H. Lindner, G. Refael, and V. Galitski, Nat. Phys. 7, 490 (2011).

[15] C. J. Fujiwara, K. Singh, Z. A. Geiger, R. Senaratne, S. V. Rajagopal, M. Lipatov, and D. M. Weld, Phys. Rev. Lett. 122, 010402 (2019).

[16] A. Alberti, V. V. Ivanov, G. M. Tino, and G. Ferrari, Nat. Phys. 5, 547 (2009).

[17] E. Haller, R. Hart, M. J. Mark, J. G. Danzl, L. Reichsöllner, and H.-C. Nägerl, Phys. Rev. Lett. 104, 200403 (2010).

[18] S. Arlinghaus and M. Holthaus, Phys. Rev. B 84, 054301 (2011).

[19] K. Kudo and T. S. Monteiro, Phys. Rev. A 83, 053627 (2011).

[20] L. Yuan and S. Fan, Optica 3, 1014 (2016).

[21] D. M. Gangardt and A. Kamenev, Phys. Rev. Lett. 102, 070402 (2009).

[22] F. Meinert, M. Knap, E. Kirilov, K. Jag-Lauber, M. B. Zvonarev, E. Demler, and H.-C. Nägerl, Science 356, 945 (2017).

[23] G. Floquet, Ann. Sci. Éc. Norm. Supér. 12, 47 (1883). 
[24] E. Ince, Ordinary Differential Equations (Dover, New York, 1956).

[25] W. Magnus and S. Winkler, Hill's Equation, Dover Books on Mathematics Series (Dover, New York, 2004).

[26] General nonlinear dispersions $\epsilon_{ \pm}(k)$, e.g., two hyperbolic branches, give rise to sequences of intersections that are not equidistant in $k$ and thereby deny a constant Floquet oscillation period.

[27] P. Hänggi, Quantum Transport and Dissipation (Wiley-VCH, Weinheim, Germany, 1998).

[28] L. Landau, Phys. Z. Sowjetunion 2, 46 (1932).

[29] C. Zener, Proc. R. Soc. London, Ser. A 137, 696 (1932).

[30] L. Landau, Phys. Z. Sowjetunion 1, 88 (1932).

[31] E. K. G. Stueckelberg, Theorie der unelastischen Stösse zwischen Atomen (Birkhäuser, Basel, 1933).

[32] B. M. Breid, D. Witthaut, and H. J. Korsch, New J. Phys. 8, 110 (2006).

[33] V. Krueckl and K. Richter, Phys. Rev. B 85, 115433 (2012).

[34] E. Schrödinger, Über die kräftefreie Bewegung in der relativistischen Quantenmechanik (Akademie der Wissenschaften in Kommission bei W. de Gruyter u. Company, Berlin, 1930).

[35] W. Zawadzki and T. M. Rusin, J. Phys.: Condens. Matter 23, 143201 (2011).

[36] V. Krückl, Wave packets in mesoscopic systems: From timedependent dynamics to transport phenomena in graphene and topological insulators, Ph.D. thesis, Universitätsverlag Regensburg, 2013 .

[37] C. Lanczos, J. Res. Natl. Bur. Stand. B 45, 255 (1950).

[38] T. Oka and H. Aoki, Phys. Rev. B 79, 081406(R) (2009).

[39] O. V. Kibis, Phys. Rev. B 81, 165433 (2010).

[40] Y. Zhou and M. W. Wu, Phys. Rev. B 83, 245436 (2011).

[41] S. E. Savel'ev and A. S. Alexandrov, Phys. Rev. B 84, 035428 (2011).

[42] J. Atteia, J. H. Bardarson, and J. Cayssol, Phys. Rev. B 96, 245404 (2017).

[43] H. K. Kelardeh, V. Apalkov, and M. I. Stockman, Phys. Rev. B 93, 155434 (2016).

[44] P. Delplace, Á. Gómez-León, and G. Platero, Phys. Rev. B 88, 245422 (2013).

[45] G. Usaj, P. M. Perez-Piskunow, L. E. F. Foa Torres, and C. A. Balseiro, Phys. Rev. B 90, 115423 (2014).

[46] P. M. Perez-Piskunow, L. E. F. Foa Torres, and G. Usaj, Phys. Rev. A 91, 043625 (2015).

[47] M. I. Katsnelson, Graphene: Carbon in Two Dimensions (Cambridge University Press, Cambridge, UK, 2012).

[48] L. Wang, I. Meric, P. Y. Huang, Q. Gao, Y. Gao, H. Tran, T. Taniguchi, K. Watanabe, L. M. Campos, D. A. Muller, J. Guo,
P. Kim, J. Hone, K. L. Shepard, and C. R. Dean, Science 342, 614 (2013).

[49] A. I. Berdyugin, S. G. Xu, F. M. D. Pellegrino, R. Krishna Kumar, A. Principi, I. Torre, M. Ben Shalom, T. Taniguchi, K. Watanabe, I. V. Grigorieva, M. Polini, A. K. Geim, and D. A. Bandurin, Science 364, 162 (2019).

[50] A. H. Castro Neto, F. Guinea, N. M. R. Peres, K. S. Novoselov, and A. K. Geim, Rev. Mod. Phys. 81, 109 (2009).

[51] M. Z. Hasan and C. L. Kane, Rev. Mod. Phys. 82, 3045 (2010).

[52] X.-L. Qi and S.-C. Zhang, Rev. Mod. Phys. 83, 1057 (2011).

[53] L. Tarruell, D. Greif, T. Uehlinger, G. Jotzu, and T. Esslinger, Nature (London) 483, 302 (2012).

[54] A treatment of Bloch oscillations in tilted honeycomb lattices can be found in A. R. Kolovsky and E. N. Bulgakov, Phys. Rev. A 87, 033602 (2013).

[55] A. R. Kolovsky, Phys. Rev. A 98, 013603 (2018).

[56] G. Weick, C. Woollacott, W. L. Barnes, O. Hess, and E. Mariani, Phys. Rev. Lett. 110, 106801 (2013).

[57] T. Jacqmin, I. Carusotto, I. Sagnes, M. Abbarchi, D. D. Solnyshkov, G. Malpuech, E. Galopin, A. Lemaitre, J. Bloch, and A. Amo, Phys. Rev. Lett. 112, 116402 (2014).

[58] S. Ganichev and W. Prettl, Intense Terahertz Excitation of Semiconductors (Oxford University Press, Oxford, UK, 2005).

[59] R. A. Lewis, J. Phys. D: Appl. Phys. 47, 374001 (2014).

[60] J. H. Shirley, Phys. Rev. 138, B979 (1965).

[61] H. Sambe, Phys. Rev. A 7, 2203 (1973).

[62] P. Reck, C. Gorini, A. Goussev, V. Krueckl, M. Fink, and K. Richter, Phys. Rev. B 95, 165421 (2017).

[63] J. Schliemann, D. Loss, and R. M. Westervelt, Phys. Rev. Lett. 94, 206801 (2005).

[64] R. Gerritsma, G. Kirchmair, F. Zahringer, E. Solano, R. Blatt, and C. F. Roos, Nature (London) 463, 68 (2010).

[65] C. Qu, C. Hamner, M. Gong, C. Zhang, and P. Engels, Phys. Rev. A 88, 021604(R) (2013).

[66] L. J. LeBlanc, M. C. Beeler, K. Jiménez-García, A. R. Perry, S. Sugawa, R. A. Williams, and I. B. Spielman, New J. Phys. 15, 073011 (2013).

[67] P. Reck, C. Gorini, and K. Richter, Phys. Rev. B 101, 094306 (2020).

[68] A. Nauts and R. E. Wyatt, Phys. Rev. Lett. 51, 2238 (1983).

[69] T. J. Park and J. C. Light, J. Chem. Phys. 85, 5870 (1986).

[70] H. Tal-Ezer and R. Kosloff, J. Chem. Phys. 81, 3967 (1984).

[71] R. Kosloff, Annu. Rev. Phys. Chem. 45, 145 (1994).

[72] M. Hochbruck and C. Lubich, SIAM J. Numer. Anal. 34, 1911 (1997). 